\title{
Symptomatic presentations of severe aortic stenosis
}

\section{Sung-Ji Park}

Division of Cardiology, Department of Internal Medicine, Cardiovascular Imaging Center, Heart Vascular Stroke Institute, Samsung Medical Center, Sungkyunkwan University School of Medicine, Seoul, Korea

Received: August 2, 2017

Revised: September 12, 2017

Accepted: September 19, 2017

Corresponding author:

Sung-Ji Park

Division of Cardiology,

Department of Internal

Medicine, Cardiovascular

Imaging Center, Heart Vascular

Stroke Institute, Samsung

Medical Center, Sungkyunkwan University School of Medicine, 81 Irwon-ro, Gangnam-gu,

Seoul 06351, Korea

Tel: +82-2-3410-3419

E-mail: tyche.park@gmail.com

\section{ABSTRACT}

Aortic stenosis (AS) is the most common type of valvular heart disease. A manifestation of ageing, the disorder is becoming more frequent as the average age of the population increases. Onset of cardinal manifestation of AS-angina, syncope, and heart failureremains the major demarcation point in the disease's course. It has been well described that patients' survival is limited once they develop symptoms from AS and survival after the onset of a symptom depends on what type of symptom a patient develops. Knowing how the pathophysiology of AS causes typical symptoms and death is paramount to understanding the disease. We discuss these issues in this review.

Keywords: Aortic valve stenosis, Chest pain, Dyspnea, Echocardiography, syncope
This is an Open Access article distributed under the terms of the Creative Commons Attribution Non-Commercial License (http:// creativecommons.org/licenses/ by-nc/4.0/).

\section{INTRODUCTION}

Aortic stenosis (AS) is the most common valvular heart disease in the elderly characterized by fixed aortic valve narrowing, left ventricular (LV) remodeling with hypertrophy, and progressive diastolic dysfunction [1]. The cardinal manifestations of AS include syncope, chest pain, and dyspnea. It has been well described that patients' survival is limited once they develop symptoms from AS and survival after the onset of a symptom depends on what type of symptom a patient develops [2]. Therefore, the onset or presence of these symptoms are the class I indication for surgical indication [3], but without any distinction among symptoms.

Is there any significant correlation between the severity of AS and development of symptoms? The development of symptoms in asymptomatic AS was predicted by aortic jet velocity at baseline, the rate of change in aortic jet velocity, and baseline functional status [4]. However, there was a large overlap between symptomatic and asymptomatic patients, consistent with the known heterogeneous response to the pressure load of AS [5]. Therefore, the occurrence and severity of cardiac symptoms may correlate poorly with the severity of AS (aortic valve area [AVA] and transvalvular pressure gradients), and symptoms often occur despite preserved LV ejection fraction (EF) $[6,7]$. 
THE EFFECT OF LEFT VENTRICULAR HYPERTROPHY ON SYMPTOMS STATUS IN AS

Hypertrophy is considered one of the major mechanisms of the myocardium for adapting to hemodynamic overload. More muscle mass provides more contractile elements for generating the extra work required by the overload. In pressure overload of AS, concentric LVH normalizes wall stress, a key determinant of ejection performance [8].

Afterload is often expressed as ventricular wall stress $(\delta)$. In this way, the high systolic pressure required to drive blood through a very stenotic aortic valve and it can be consistent with normal afterload and normal EF. Since afterload is a key determinant of ejection performance, its normalization is important in maintaining normal EF and stroke volume. LV pressure overload may induce LV hypertrophy. Unfortunately, hypertrophy is a double-edged sword. Although it helps to preserve ejection performance, hypertrophy also impairs coronary blood flow reserve inducing myocardial fibrosis, which in turn leads to increased chamber stiffness, delayed active LV relaxation, and compromised coronary flow reserve. All of these changes may affect diastolic and systolic function and be associated with increased mortality $[9,10]$. The magnitude of LVH did not by itself explain the presentations of symptoms of AS [7]. Pressure gradient nor AVA are not predictive of symptom onset because those factors alone do not by themselves control the myocardium's response to AS [11]. It is thus possible that the development of symptoms in AS reflects alterations in LV morphology and function, leading to increased filling pressures and left atrial (LA) dilatation, hemodynamic, and morphological changes that may occur without any change in LVEF.

\section{ASYMPTOMATIC VERSUS SYMPTOMATIC AS}

The basic question is which echocardiographic parameters differentiate the presence of symptoms in severe AS?

The hemodynamic consequence of AS is LV pressure overload, causing morphological changes of the LV characterized by LV hypertrophy, concentric remodeling, and myocardial fibrosis. As a consequence of increased afterload [8] and LV
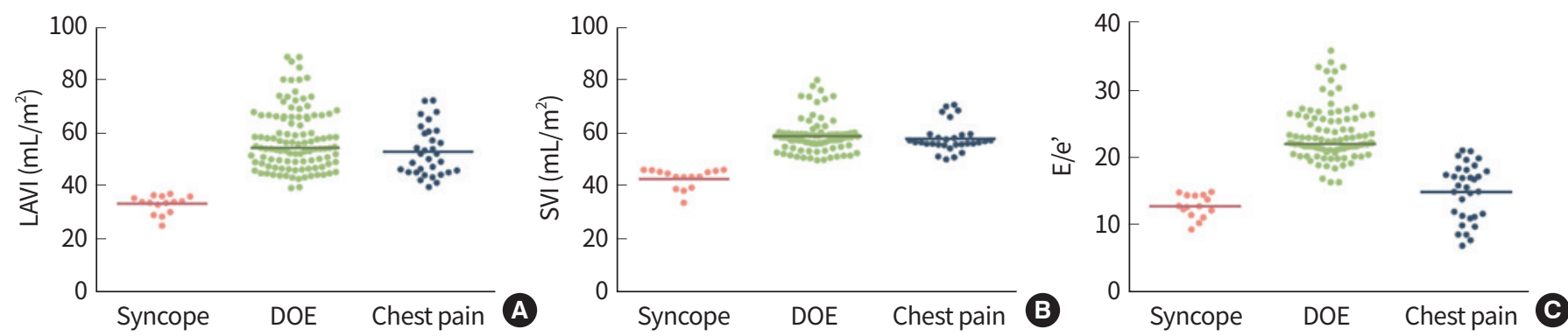

Fig. 1. (A) Left atrial volume index (LAVi), (B) stroke volume index (SVI), and (C) E/e' ratio in patients with different symptoms. Adapted from Park et al. [7]. DOE, dyspnea on exertion.
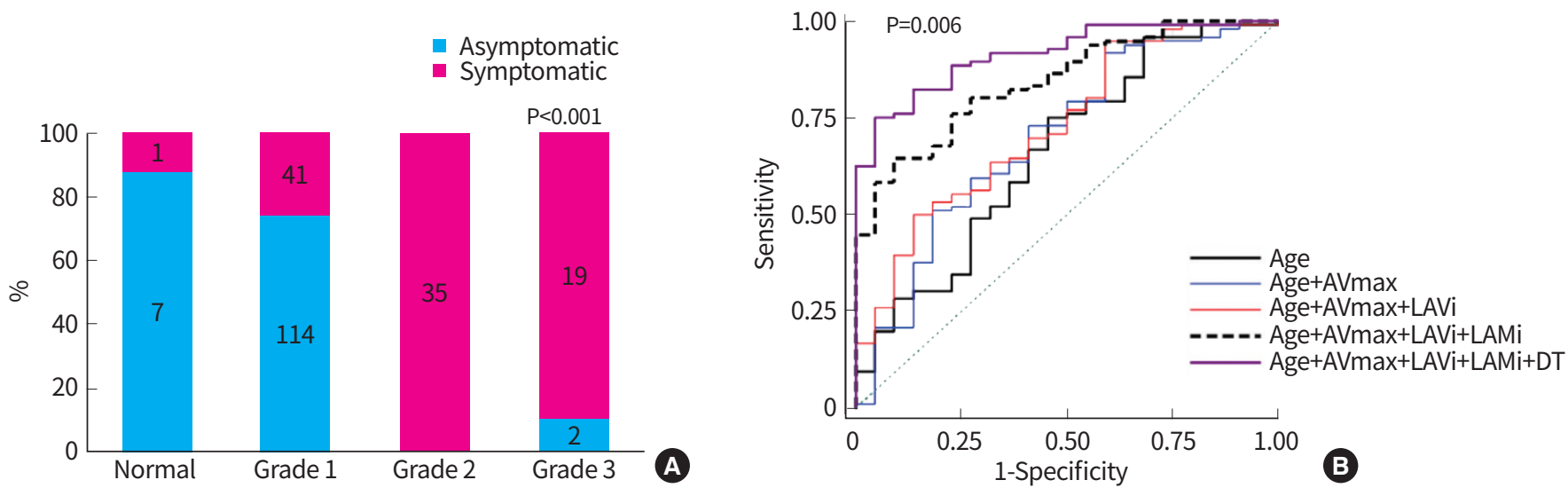

Fig. 2. (A) Symptomatic status as a function of diastolic grade, (B) Incremental information of age, maximum aortic valve velocity (AVmax), left atrial volume index (LAVi), LVMi, and deceleration time in predicting symptom status. Adapted from Dahl et al., with permission from Wolters Kluwer Health [17]. 
remodeling [12-14], AS will lead to LV diastolic dysfunction, increased filling pressures, and heart failure (HF) symptoms. The development of HF symptoms has previously been demonstrated to be associated with measures of AS severity and LV hypertrophy [15,16]. In contrasts, Park et al. [7] recently demonstrated that compared with the asymptomatic group, symptomatic patients were older and had lower cardiac output, and higher E/e' ratio while having a similar AVA and gradient in 498 severe AS and normal LVEF. Syncope group displayed smaller LV dimension, stroke volume, cardiac output, left atrial volume index (LAVi), and E/e' ratio. Conversely, dyspnea group was found to have the worst diastolic function with largest LAVi and highest E/e' ratio (Fig. 1). Dahl et al. [17] also compared clinical characteristics and echocardiographic parameters in 99 symptomatic and 139 asymptomatic patients with severe AS and normal LVEF. This study demonstrated that symptomatic status was associated with $\mathrm{LVH}$, concentric remodeling, diastolic dysfunction, and dilatation of LA. An LV diastolic grade of 2 or 3 was highly associated with the presence of symptoms and model including age, maximum aortic valve velocity, left ventricular mass index (LVMi), LAVi, and deceleration time provided incremental information on symptomatic status (Fig. 2).

\section{DYSPNEA}

Onset of dyspnea and other symptoms of HF presage the worst outlook for the patient with AS [18]. The first question is what causes the dyspnea in severe AS? In the patients with severe AS who develop dyspnea, markedly altered LV diastolic function with increased filling pressure was present (Fig. 1) [7]. Left-sided filling pressures seem to have a good correlation with the symptom [19]. Diastolic dysfunction, which increases LV filling pressure, predicted dyspnea in severe AS patients. The second question is what causes the diastolic dysfunction in severe AS? Diastole is typically divided into two parts, early active relaxation and passive filling [10]. Early relaxation is described by LV pressure decay time, whereas passive filling is characterized best by the diastolic pressure-volume relationship of the LV. During active relaxation, calcium is pumped back into the sarcoplasmic reticulum, causing the contractile interaction between actin and myosin to diminish. In pathologic LVH, early active relaxation is delayed, reflecting abnormalities in the calcium handling that pumps calcium back into the sarcoplasmic reticulum, reducing actin-myosin interaction [20]. This slowing of active relaxation delays the diastolic fall in LV pressure during isovo- lumic relaxation, in turn delaying mitral valve opening and shortening the time for the LV to fill. Passive filling in pathologic LVH is characterized by a shift in the diastolic pressure-volume relation of the LV upward and leftward, thus requiring increased filling pressure to fill the LV to any given volume because the LV is stiffer than normal. Increased LV stiffness in pathologic LVH accrues both from intrinsic and extrinsic factors. Intrinsically, myocytes are thicker and filled with a denser than normal cytoskeleton [21], in turn causing the myocyte to be stiffer than normal. Increased chamber stiffness is also due to increased extracellular components including the collagen network that holds the LV together and that connects the myocyte to the chamber [22]. In severe AS patient, at least four factors - LV thickness, active relaxation, myocyte structure, and extracellular matrix-can vary from patient to patient. Those four factors can alter diastolic function [11]. This complexity and augmented diastolic dysfunction leads to pulmonary congestion and dyspnea in severe AS.

\section{SYNCOPE}

Syncope is one of the most urgent clinical symptoms, occurring suddenly without warning. Syncope occurs when there is inadequate systemic blood pressure to support cerebral blood flow. Three mechanisms have been suggested to explain exertional syncope in patients with AS: (1) cardiac arrhythmias, (2) sudden failure of an overloaded LV during the stress of exercise, (3) peripheral vasodilatation occurring suddenly and inappropriately in the face of fixed cardiac output [23]. Inappropriate vasodilation, the result of reflexes triggered by LV baroreceptors [24] and occurring during or just after exercise, is thought to be the mechanism in most cases [25]. The exact mechanism of syncope in AS still remains unclear.

The prevalence of AS has increased with aging. The main cause has markedly changed from rheumatic pathogenesis to arteriosclerosis [26]. Almost all current clinical studies of AS discuss patients aged $>70$ years $[15,27,28]$, contrary to the average age of 48 years at clinical presentation reported by Ross and Braunwald [2]. It is natural to think that the perception from clinical studies of the past would not apply to the new condition in recent years. However, only few recent studies have investigated on syncope in patients with AS $[25,29,30]$.

Park et al. [7] demonstrated that the group with syncope had the lowest stroke volume along with the smallest LV 


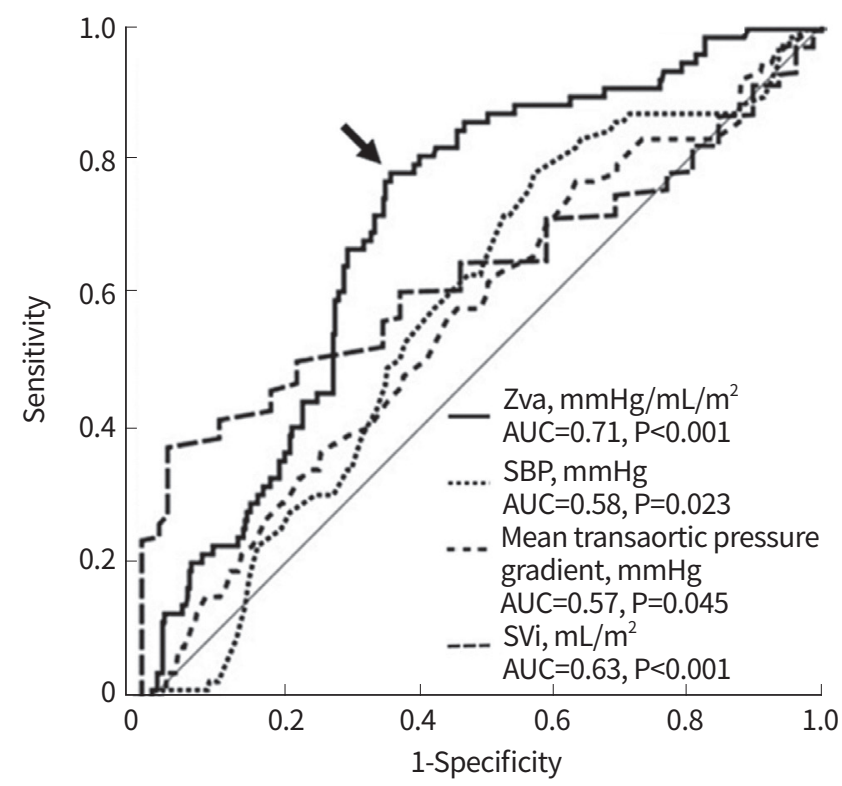

Fig. 3. Receiver operating characteristic curve analysis of the ability of valvuloarterial impedance (Zva), systolic blood pressure (SBP), mean transaortic pressure gradient, and stroke volume index (SVi) to predict syncope in patients with aortic stenosis. Adapted from Harada et al., with permission from Wolters Kluwer Health [31]. AUC, area under the curve.

mass and LV volumes and LA volume. Additionally E/e', a guide to filling pressure, was also lowest in this syncope group. These data suggest that patients with syncope have remodeled in such a way as to have smaller hearts that generate less cardiac output, possibly compounded by lower LV filling pressure further limiting cardiac output [11].

The important question is which echocardiographic parameters predict the development of syncope in severe AS. Recently, Harada et al. [31] suggests that valvuloarterial impedance $\geq 4.7 \mathrm{~mm} \mathrm{Hg} / \mathrm{mL}$ per $\mathrm{m}^{2}$ may have clinical significance because it may represent not only the cutoff value of syncope prediction, but also an afterload level that surpasses the capabilities of the LV compensatory mechanism. In this study, conventional parameters of AS severity, including mean transaortic pressure gradient and AVA, were unable to independently predict syncope (Fig. 3).

\section{ANGINA}

The incidence of angina pectoris in patients with AS has been reported to range between $30 \%$ and $40 \%$ in the absence of associated obstructive coronary artery disease [32-35]. The pathophysiological mechanism of angina is not clear but seems to be due to unbalanced myocardial oxygen supply and demand. Oxygen demand is proportional to heart rate and systolic wall stress, and the latter can be elevated in cases of AS when hypertrophy is inadequate to normalize stress [8]. After aortic valve replacement (AVR), there is marked regression of hypertrophy that may occur over the next several months to years [36], but angina is relieved immediately. Relief of angina immediately after AVR is probably due to the combination of sudden decreased oxygen demand after removal of pressure overload and increased oxygen supply of improved perfusion [37].

On the oxygen supply side, it is well known that coronary flow reserve is reduced in AS patients [38]. This phenomenon has been revealed by invasive coronary catheterization [38] and imaging modalities such as positron emission tomography [39] and cardiac magnetic resonance (CMR) [40,41]. Julius et al. [42] demonstrated patients with angina had a lower LV muscle mass, an increased LV peak systolic pressure, and increased wall stress than those without angina. Vessels of the left coronary artery were smaller and coronary flow reserve was lower in patients with angina than in those without angina. Inadequate LV hypertrophy with an increased wall stress was found in patients with angina but not in patients without angina. However, there is no difference in the flow reserve of patients with versus those without angina. Thus, this factor must in some way contribute to the potential for ischemia to develop in severe AS.

Myocardial ischemia in patients with severe AS can occur in the absence of coronary artery disease and appears to be due to inadequate LVH with high systolic and diastolic wall stresses and a somehow reduced coronary flow reserve.

In the absence of significant coronary stenosis, this finding is indicative of microvascular dysfunction, but it remains unclear whether the reduced myocardial perfusion reserve seen in severe AS without obstructive coronary artery disease (CAD) leads to angina during stress stimuli.

Adenosine-stress CMR can detect stress-induced abnormal hypoperfusion with signs and symptoms of ischemia without CAD $[43,44]$ and this is the almost the only noninvasive clinical method that allows assessment of the transmyocardial distribution of coronary blood flow and myocardial perfusion reserve index. Among severe AS patients with angina but no obstructive CAD, Park et al. [7] demonstrated a reduced myocardial perfusion reserve, which is indicative of microvascular dysfunction, compared with severe AS patients without any symptoms [45]. Park et al. [7] suggests that angina in patients with severe AS without obstructive CAD might be attributed to $\mathrm{LVH}$, which can cause myocardial ischemia by coronary microvascular dysfunction (Fig. 4). 


\section{CENTRAL ILLUSTRATION Coronary Microvascular Dysfunction: Mechanism of Occurrence of Angina}

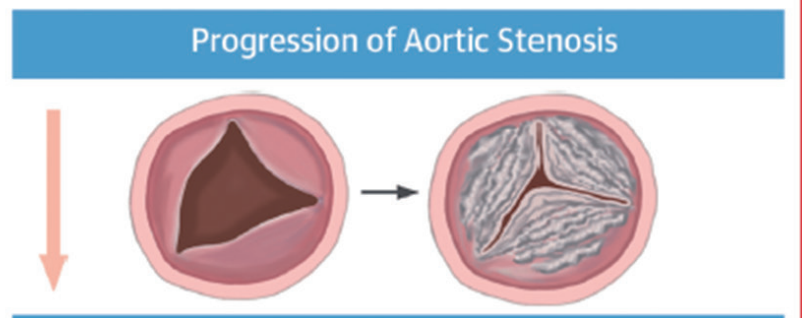

Development of Left Ventricular Hypertrophy
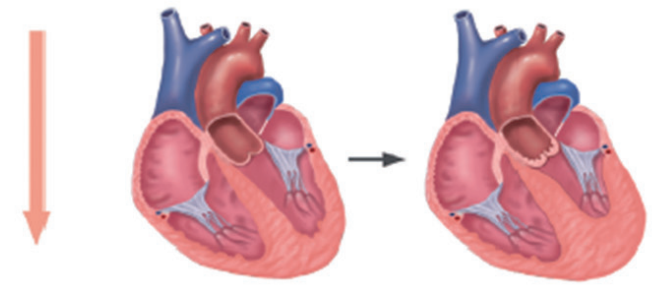

Decrement of MPRI

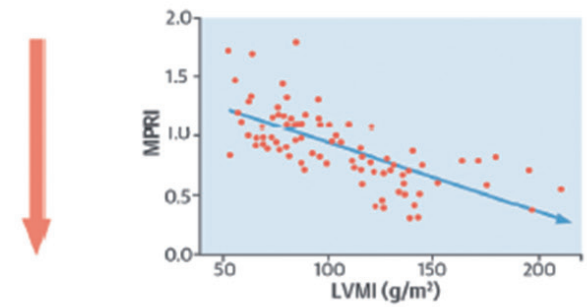

Occurrence of Angina

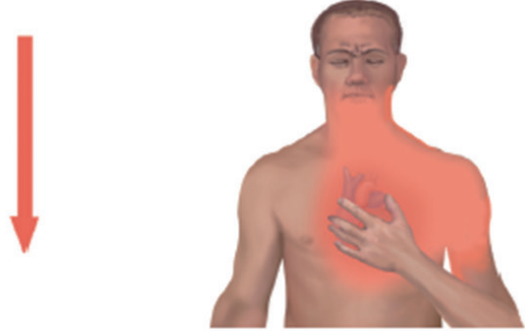

Ahn, J.-H. et al. J Am Coll Cardiol. 2016; 67(12):1412-22.

In patients with severe aortic stenosis (AS) without obstructive coronary artery disease, angina is related to the decrement of MPRI (impaired coronary microvascular function) along with development of left ventricular hypertrophy (LVH). LVMI = left ventricular mass index; MPRI = myocardial perfusion reserve index.

Fig. 4. Coronary microvascular dysfunction: mechanism of occurrence of angina. Adapted from Ahn et al. [45]. MPRI, myocardial perfusion reserve index.

\section{CONCLUSION}

Diastolic dysfunction is important in the natural history and its role in presenting symptoms of patients with severe AS. In patients with normal EF, diastolic dysfunction is a key driver of development dyspnea. Smaller LV volumes reduce cardiac output, predisposing the patient to syncope. Angina can occur in the absence of coronary artery disease and appears to be due to inadequate LVH with high systolic and diastolic wall stresses and a somehow reduced coronary flow reserve. Future prospective studies should be conducted to clarify the pathophysiologic mechanisms of presentation of specific symptoms in severe AS.

\section{CONFLICTS OF INTEREST}

No potential conflict of interest relevant to this article was reported.

\section{REFERENCES}

1. Kennedy KD, Nishimura RA, Holmes DR Jr, Bailey KR. Natural history of moderate aortic stenosis. J Am Coll Cardiol 1991;17:313-9.

2. Ross J Jr, Braunwald E. Aortic stenosis. Circulation 1968; 38(1 Suppl):61-7.

3. Nishimura RA, Otto CM, Bonow RO, Carabello BA, Erwin JP 3rd, Guyton RA, et al. 2014 AHA/ACC guideline for the management of patients with valvular heart disease: executive summary: a report of the American College of Cardiology/American Heart Association Task Force on Practice Guidelines. J Am Coll Cardiol 2014;63:2438-88.

4. Otto CM, Burwash IG, Legget ME, Munt BI, Fujioka M, Healy NL, et al. Prospective study of asymptomatic valvular aortic stenosis. Clinical, echocardiographic, and exercise predictors of outcome. Circulation 1997;95:2262-70.

5. Otto CM. Aortic stenosis: listen to the patient, look at the valve. N Engl J Med 2000;343:652-4.

6. Vahanian A, Baumgartner H, Bax J, Butchart E, Dion R, Filippatos $\mathrm{G}$, et al. Guidelines on the management of valvular heart disease: the Task Force on the Management of Valvular Heart Disease of the European Society of Cardiology. Eur Heart J 2007;28:230-68.

7. Park SJ, Enriquez-Sarano M, Chang SA, Choi JO, Lee SC, Park SW, et al. Hemodynamic patterns for symptomatic presentations of severe aortic stenosis. JACC Cardiovasc Imaging 2013;6:137-46.

8. Grossman W, Jones D, McLaurin LP. Wall stress and patterns of hypertrophy in the human left ventricle. J Clin Invest 1975;56:56-64.

9. Levy D, Garrison RJ, Savage DD, Kannel WB, Castelli WP. 
Prognostic implications of echocardiographically determined left ventricular mass in the Framingham Heart Study. N Engl J Med 1990;322:1561-6.

10. Zile MR, Brutsaert DL. New concepts in diastolic dysfunction and diastolic heart failure: Part I: diagnosis, prognosis, and measurements of diastolic function. Circulation 2002;105:1387-93.

11. Carabello BA. The symptoms of aortic stenosis: a step closer to understanding their cause. JACC Cardiovasc Imaging 2013;6:147-9.

12. Kupari M, Turto H, Lommi J. Left ventricular hypertrophy in aortic valve stenosis: preventive or promotive of systolic dysfunction and heart failure? Eur Heart J 2005;26: 1790-6.

13. Ikonomidis I, Tsoukas A, Parthenakis F, Gournizakis A, Kassimatis A, Rallidis L, et al. Four year follow up of aortic valve replacement for isolated aortic stenosis: a link between reduction in pressure overload, regression of left ventricular hypertrophy, and diastolic function. Heart 2001;86:309-16.

14. Orsinelli DA, Aurigemma GP, Battista S, Krendel S, Gaasch WH. Left ventricular hypertrophy and mortality after aortic valve replacement for aortic stenosis: a high risk subgroup identified by preoperative relative wall thickness. J Am Coll Cardiol 1993;22:1679-83.

15. Pellikka PA, Sarano ME, Nishimura RA, Malouf JF, Bailey $\mathrm{KR}$, Scott CG, et al. Outcome of 622 adults with asymptomatic, hemodynamically significant aortic stenosis during prolonged follow-up. Circulation 2005;111:32905.

16. Monrad ES, Hess OM, Murakami T, Nonogi H, Corin WJ, Krayenbuehl HP. Abnormal exercise hemodynamics in patients with normal systolic function late after aortic valve replacement. Circulation 1988;77:613-24.

17. Dahl JS, Christensen NL, Videbaek L, Poulsen MK, Carter-Storch R, Hey TM, et al. Left ventricular diastolic function is associated with symptom status in severe aortic valve stenosis. Circ Cardiovasc Imaging 2014;7:142-8.

18. Carabello BA, Paulus WJ. Aortic stenosis. Lancet 2009; 373:956-66.

19. Solomonica A, Burger AJ, Aronson D. Hemodynamic determinants of dyspnea improvement in acute decompensated heart failure. Circ Heart Fail 2013;6:53-60.

20. Zile MR, Baicu CF, Gaasch WH. Diastolic heart failure: abnormalities in active relaxation and passive stiffness of the left ventricle. N Engl J Med 2004;350:1953-9.

21. Cooper G 4th. Cardiocyte cytoskeleton in hypertrophied myocardium. Heart Fail Rev 2000;5:187-201.

22. Weber KT, Brilla CG. Pathological hypertrophy and cardiac interstitium: fibrosis and renin-angiotensin-aldosterone system. Circulation 1991;83:1849-65.

23. Schwartz LS, Goldfischer J, Sprague GJ, Schwartz SP. Syncope and sudden death in aortic stenosis. Am J Cardiol 1969;23:647-58.

24. Johnson AM. Aortic stenosis, sudden death, and the left ventricular baroceptors. Br Heart J 1971;33:1-5.

25. Richards AM, Nicholls MG, Ikram H, Hamilton EJ, Richards RD. Syncope in aortic valvular stenosis. Lancet 1984;2: 1113-6.

26. Lindroos M, Kupari M, Heikkila J, Tilvis R. Prevalence of aortic valve abnormalities in the elderly: an echocardiographic study of a random population sample. J Am Coll Cardiol 1993;21:1220-5.

27. Hachicha Z, Dumesnil JG, Bogaty P, Pibarot P. Paradoxical low-flow, low-gradient severe aortic stenosis despite preserved ejection fraction is associated with higher afterload and reduced survival. Circulation 2007;115:2856-64.

28. Herrmann S, Stork S, Niemann M, Lange V, Strotmann JM, Frantz $\mathrm{S}$, et al. Low-gradient aortic valve stenosis myocardial fibrosis and its influence on function and outcome. J Am Coll Cardiol 2011;58:402-12.

29. Wilmshurst PT, Willicombe PR, Webb-Peploe MM. Effect of aortic valve replacement on syncope in patients with aortic stenosis. Br Heart J 1993;70:542-3.

30. Omran H, Fehske W, Rabahieh R, Hagendorff A, Pizzulli L, Zirbes M, et al. Valvular aortic stenosis: risk of syncope. J Heart Valve Dis 1996;5:31-4.

31. Harada K, Saitoh T, Tanaka J, Shibayama K, Berdejo J, Shiota T. Valvuloarterial impedance, but not aortic stenosis severity, predicts syncope in patients with aortic stenosis. Circ Cardiovasc Imaging 2013;6:1024-31.

32. Basta LL, Raines D, Najjar S, Kioschos JM. Clinical, haemodynamic, and coronary angiographic correlates of angina pectoris in patients with severe aortic valve disease. Br Heart J 1975;37:150-7.

33. Hakki AH, Kimbiris D, Iskandrian AS, Segal BL, Mintz GS, Bemis CE. Angina pectoris and coronary artery disease in patients with severe aortic valvular disease. Am Heart J 1980;100:441-9.

34. Mandal AB, Gray IR. Significance of angina pectoris in aortic valve stenosis. Br Heart J 1976;38:811-5.

35. Nadell R, DePace NL, Ren JF, Hakki AH, Iskandrian AS, Morganroth J. Myocardial oxygen supply/demand ratio in aortic stenosis: hemodynamic and echocardiographic 
evaluation of patients with and without angina pectoris. J Am Coll Cardiol 1983;2:258-62.

36. Monrad ES, Hess OM, Murakami T, Nonogi H, Corin WJ, Krayenbuehl HP. Time course of regression of left ventricular hypertrophy after aortic valve replacement. Circulation 1988;77:1345-55.

37. Gould KL, Carabello BA. Why angina in aortic stenosis with normal coronary arteriograms? Circulation 2003; 107:3121-3.

38. Marcus ML, Doty DB, Hiratzka LF, Wright CB, Eastham CL. Decreased coronary reserve: a mechanism for angina pectoris in patients with aortic stenosis and normal coronary arteries. N Engl J Med 1982;307:1362-6.

39. Rajappan K, Rimoldi OE, Dutka DP, Ariff B, Pennell DJ, Sheridan DJ, et al. Mechanisms of coronary microcirculatory dysfunction in patients with aortic stenosis and angiographically normal coronary arteries. Circulation 2002; 105:470-6.

40. Steadman CD, Jerosch-Herold M, Grundy B, Rafelt S, Ng LL, Squire IB, et al. Determinants and functional significance of myocardial perfusion reserve in severe aortic stenosis. JACC Cardiovasc Imaging 2012;5:182-9.
41. Mahmod M, Francis JM, Pal N, Lewis A, Dass S, De Silva R, et al. Myocardial perfusion and oxygenation are impaired during stress in severe aortic stenosis and correlate with impaired energetics and subclinical left ventricular dysfunction. J Cardiovasc Magn Reson 2014;16:29.

42. Julius BK, Spillmann M, Vassalli G, Villari B, Eberli FR, Hess OM. Angina pectoris in patients with aortic stenosis and normal coronary arteries. Mechanisms and pathophysiological concepts. Circulation 1997;95:892-8.

43. Lanza GA, Buffon A, Sestito A, Natale L, Sgueglia GA, Galiuto $L$, et al. Relation between stress-induced myocardial perfusion defects on cardiovascular magnetic resonance and coronary microvascular dysfunction in patients with cardiac syndrome X. J Am Coll Cardiol 2008;51:466-72.

44. Panting JR, Gatehouse PD, Yang GZ, Grothues F, Firmin $\mathrm{DN}$, Collins $\mathrm{P}$, et al. Abnormal subendocardial perfusion in cardiac syndrome $X$ detected by cardiovascular magnetic resonance imaging. N Engl J Med 2002;346:1948-53.

45. Ahn JH, Kim SM, Park SJ, Jeong DS, Woo MA, Jung SH, et al. Coronary microvascular dysfunction as a mechanism of angina in severe AS: prospective adenosine-stress CMR study. J Am Coll Cardiol 2016;67:1412-22. 